\title{
Enhancing Patient Outcomes with Clinical Nutrition: The Effects of Supplementation in Orthopedics
}

\author{
Alexa Abdelaziz*1, Ralph Venuto ${ }^{2}$, Ryan Stahl ${ }^{3}$, John Karl OPTA ${ }^{4}$ and Sheraz Syed ${ }^{5}$ \\ ${ }^{1}$ Nutritional \& Metabolic Biology, Director of Research and Development at MEND, Columbia University, New York, USA \\ ${ }^{2}$ Orthopedic Surgeon in Newport Beach, California \\ ${ }^{3}$ PT, DPT, OCS- Ivy Rehab Physical Therapy \\ ${ }^{4}$ EXOS XPS Sports Performance Specialist, CES, BMS - Ivy Rehab Physical Therapy \\ ${ }^{5}$ Physical Therapist Cert. MDT - Triumph Physical Therapy
}

*Corresponding author: Alexa Abdelaziz, Nutritional \& Metabolic Biology, Director of Research and Development at MEND,

Columbia University, New York, USA

\section{ARTICLE INFO}

Received: 慧 October 27, 2021

Published: 慧 November 02, 2021

Citation: Alexa Abdelaziz, Ralph Venuto, Ryan Stahl, John Karl OPTA, Sheraz Syed. Enhancing Patient Outcomes with Clinical Nutrition: The Effects of Supplementation in Orthopedics. Biomed J Sci \& Tech Res 39(4)-2021. BJSTR. MS.ID.006348.

\section{ABSTRACT}

A growing body of clinical evidence suggests that orthopedic patient outcomes can be enhanced through supplementation with amino acids, protein and other natural compounds. This paper reviews the state of the clinical evidence, the regulatory landscape for physical therapy to incorporate nutrition into clinical practice and the potential cost savings and economic benefit to society.

Keywords: Nutrition; Orthopedics; Outcomes; Supplementation; Healing; Recovery

\section{Introduction}

Each year more than half of people over the age of 18 in the US will develop a musculoskeletal injury that lasts more than 3 months. This is roughly $18 \%$ of all clinical visits and represents enormous costs, $\sim 5.7 \%$ of US GDP and 216 million lost workdays [1]. Tens of millions of patients each year turn to orthopedic surgeons and physical therapists to treat their injuries and return to normal function as quickly as possible. There is an urgent need to utilize all of the latest techniques, tools and technologies to improve outcomes and enhance patient recovery to lower the cost burden on the health system and improve economic output due to lost workdays. New innovations continue to enhance the field of musculoskeletal injury treatment and management. One area that is showing considerable promise is in targeted nutrition.

\section{Nutrition and Healing}

Science is increasingly showing just how critical nutrition is to healing and recovery. As we know during a state of trauma, such as injury or surgery, the body's nutritional needs increase:

a) The body enters a higher metabolic state and requires more energy

b) Trauma and lack of use leads to muscle atrophy, which prolongs recovery 
c) The immune system is weakened due to stress and shock

d) Risk of wound infection is increased

e) Persistent inflammation delays return of function

f) Trauma and physiological stress lead to increased fatigue

Nutritional deficiencies impede the natural progression of healing, including elevating the risk of infection and lengthening recovery periods. A patient that is nutritionally optimized will heal better and faster and have better long-term outcomes. One that isn't will heal more slowly and may have long-term complications. Unfortunately, most Americans are overfed and undernourished, meaning most Americans are not at optimal nutritional status to prevent complications post-trauma. Hospital studies have shown that as many as $50 \%$ of patients are undernourished or malnourished [2]. These patients face greater complications than properly nourished patients, including longer hospital stays, greater risk of infection, and increased mortality. One study, published in the Journal of Nutrition, studied over 16,000 individuals and found that many are not meeting the minimum recommended thresholds for micronutrient intake (Table 1): The combination of increased baseline nutritional needs post-trauma, from injury or surgery, and prevalent undernourishment means that most Americans are not well equipped nutritionally to heal Figure 1.

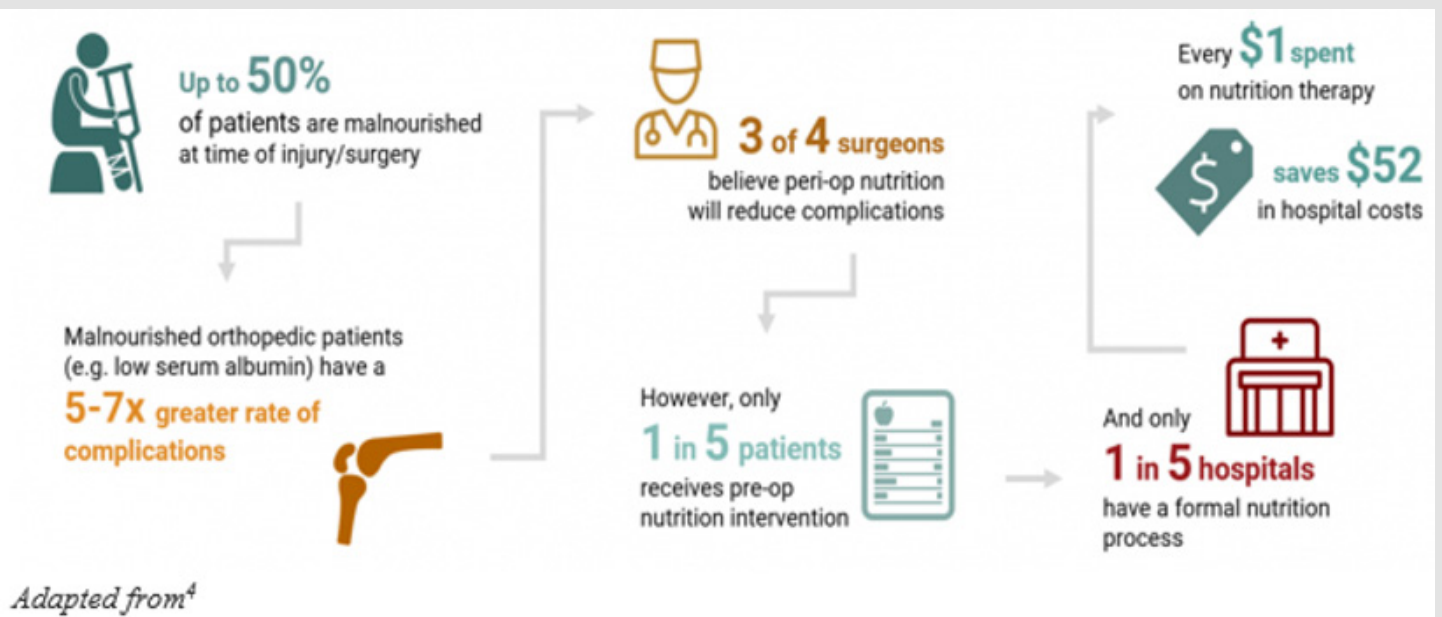

Figure 1: The Impact of Malnourishment on Healing ().

Table 1.

\begin{tabular}{|c|c|c|c|}
\hline Nutrient & \% of Americans Deficient & Nutrient & \% of Americans Deficient \\
\hline Vitamin D & $93 \%$ & Calcium & $49 \%$ \\
\hline Vitamin E & $91 \%$ & Vitamin A & $45 \%$ \\
\hline Magnesium & $55 \%$ & Vitamin C & $37 \%$ \\
\hline
\end{tabular}

Adapted from ${ }^{3}$

\section{Clinical Evidence - Nutrition as an Orthopedic Treatment Tool}

The science of nutrition in orthopedics is advancing rapidly and a growing body of clinical trials are demonstrating convincingly that targeted nutrition can enhance outcomes, both for acute patients as well as for patients with chronic conditions. Wound healing, inflammation response, increasing muscle mass and strength, and decreasing muscle atrophy are crucial recovery objectives for orthopedic patients, and nutrition has been shown to support these healing processes. Below we look at a sampling of randomized trials from a larger set of published clinical trials in orthopedics. A randomized controlled study by Ekinci et al. [3]. included 75 older female patients with hip fractures and investigated the effects of Calcium HMB, vitamin D, and protein supplementation on wound healing and muscle strength. The study group received an enteral product containing $3 \mathrm{~g}$ CaHMB, $1000 \mathrm{IU}$ vitamin D, and $36 \mathrm{~g}$ protein, in addition to standard postoperative nutrition. They found that the patients on the nutritional supplement product had an acceleration of wound healing, shortening of immobilization period, and increased muscle strength without changing body mass index. This study also found a reduced dependence to bed and related complications after an orthopedic operation [4,5]. A study by 
Negro et al. found that twice daily consumption of a mix containing Essential Amino Acids (EAA), creatine, vitamin D and Muscle Restore Complex (MRC*: Alpha Lipoic Acid (ALA), Coenzyme Q10 (CoQ10), resveratrol) for 12 weeks may aid in sarcopenia prevention without physical exercise by improving muscle aging-related outcomes, such as muscle mass, muscle strength and muscle power. In this study 38 healthy elderly subjects were randomized and allocated into the supplement or placebo group. Significant improvements were found in the supplement group compared to placebo in vitamin D blood levels, Legs Fat Free Mass, Appendicular Lean Mass, Maximal Voluntary Contraction, and Peak Power [6]. Dreyer et al. found in a double-blind, placebo-controlled, randomized trial on patients undergoing total knee arthroplasty (TKA), that EAA supplementation is safe and reduced the loss of muscle volume in older adults recovering from TKA [7]. These studies emphasize the importance of targeted nutritional supplements for muscle preservation and return to function - critical in any patient with a surgery that results in significant muscle atrophy such as ACL.

Certain key ingredients are crucial to include to help support recovery. Liberman et al. found that thirteen weeks of nutritional supplementation with Vitamin D and leucine-enriched whey protein may attenuate the progression of chronic low- grade inflammatory profile in older sarcopenic persons with mobility limitations [8]. Another key study by Kim et al. found that in surgical patients, the addition of glutamine supplementation reduced infection rates and shortened the length of hospital stay. Glutamine also decreased the production of pro-inflammatory cytokines in this population [9]. By lowering inflammation, the healing process is greatly enhanced. $\beta$-hydroxy $\beta$-methylbutyrate (HMB) has been shown in many studies to promote wound healing and diminish muscle wasting Flakoll et al. found that elderly women treated with a nutritional supplement containing HMB, arginine, and lysine for 12 weeks had increased muscle mass and maximum strength [10]. HMB is also utilized and useful in combination therapies. This doubleblind controlled 12- month study by Rathmacher et al. found that HMB in combination with Vitamin D had a significant benefit on lean body mass and showed improvement in knee extension peak torque even with no exercise. Overall, their findings showed that even without exercise, the HMB+ Vitamin D supplemented group showed significant increases in functional outputs than those in controls [11]. Interestingly, HMB has also been shown to increase anabolic signaling [12].

\section{Conclusions}

A very significant proportion of the orthopedic patient population is nutritionally compromised and during trauma the body's nutritional needs increase above baseline. Clinical studies are increasingly demonstrating that a patient's nutritional status can directly impact outcomes and that modification through supplementation can enhance outcomes. The American Physical Therapy Association (APTA) has recognized the important role of nutrition in patient care and treatment and put it in scope of practice. APTA states: "Nutrition is part of the professional scope of practice for physical therapists"; further they state: "it is the role of the physical therapist to screen for and provide information on diet and nutritional issues to patients, clients, and the community within the scope of physical therapist practice." (House of Delegates P06-15-22-17)." There remains work to be done to quantify the economic impact and savings to the healthcare system, but we suspect it is considerable. For example, large retrospective studies done by Novartis and Eli Lilly of more than 130 thousand patients shows that severe muscle atrophy and weakness (MAW) is common in joint replacement patients and that complications related to MAW cost roughly $\$ 10 \mathrm{~K}$ per patient to treat 13 . Similarly, according to a study published by Mackenzie et al. [13]. in the Journal of Orthopedic Sports Medicine, revision costs in ACL patients range roughly in the $\$ 9 \mathrm{~K}$ range [14]. Considering the sheer volume of orthopedic injuries and surgeries, we can extrapolate that there is billions of dollars of cost in the healthcare system that can be addressed through nutritional supplementation and optimization.

In summary, an increasing body of science suggests that targeted supplementation should be utilized in patient care. By doing so, we can improve patient outcomes and reduce healthcare costs.

\section{References}

1. BMUS: The Burden of Musculoskeletal Diseases in the United States (boneandjointburden.org).

2. Kirkland LL, Kashiwagi DT, Brantley S, Scheurer D, Varkey P, et al. (2013) Nutrition in the hospitalized patient. J Hosp Med 8(1): 52-58.

3. Victor L Fulgoni III, Debra R Keast, Regan L Bailey, Johanna Dwyer (2011) Foods, Fortificants, and Supplements: Where Do Americans Get Their Nutrients? The Journal of Nutrition 141(10): 1847-1854.

4. Wischmeyer PE, Carli F, Evans DC, Guilbert S, Kozar R, et al. (2018) American Society for Enhanced Recovery and Perioperative Quality Initiative Joint Consensus Statement on Nutrition Screening and Therapy Within a Surgical Enhanced Recovery Pathway. Anesth Analg 126(6): 1883-1895.

5. Ekinci O, Yanık S, Terzioğlu Bebitoğlu B, Yılmaz Akyüz E, Dokuyucu $A$, et al. (2016) Effect of Calcium $\beta$-Hydroxy- $\beta$-Methylbutyrate (CaHMB), Vitamin D, and Protein Supplementation on Postoperative Immobilization in Malnourished Older Adult Patients with Hip Fracture: A Randomized Controlled Study. Nutr Clin Pract 31(6): 829-835.

6. Negro M, Perna S, Spadaccini D, Castelli L, Calanni L, et al. (2019) Effects of 12 Weeks of Essential Amino Acids (EAA)-Based Multi-Ingredient Nutritional Supplementation on Muscle Mass, Muscle Strength, Muscle Power and Fatigue in Healthy Elderly Subjects: A Randomized Controlled Double-Blind Study. J Nutr Health Aging 23(5): 414-424. 
7. Dreyer HC, Owen EC, Strycker LA, Smolkowski K, Muyskens JB, et al. (2018) Essential Amino Acid Supplementation Mitigates Muscle Atrophy After Total Knee Arthroplasty: A Randomized, Double-Blind, PlaceboControlled Trial. JB JS Open Access 3(2): e0006.

8. Liberman K, Njemini R, Luiking Cederholm T, Bautmans I (2019) Thirteen weeks of supplementation of vitamin D and leucine- enriched whey protein nutritional supplement attenuates chronic low-grade inflammation in sarcopenic older adults: the PROVIDE study. Aging clinical and experimental research 31(6): 845-854.

9. Kim Hyeyoung (2011) "Glutamine as an immunonutrient." Yonsei medical journal 52(6): 892-897.

10. Flakoll P, Sharp R, Baier S, Deanna Levenhagen, Christopher Carr, et al (2004) Effect of betahydroxy-betamethylbutyrate, arginine, and lysine supplementation on strength, functionality, body composition, and protein metabolism in elderly women. Nutrition 20(5): 445-451.

11. Rathmacher JA, Pitchford LM, Khoo P, Angus H, Lang J, et al. (2020) Long-term Effects of Calcium $\beta$-Hydroxy- $\beta$-Methylbutyrate and Vitamin

\section{ISSN: 2574-1241}

DOI: 10.26717/BJSTR.2021.39.006348

Alexa Abdelaziz. Biomed J Sci \& Tech Res

cC (P) This work is licensed under Creative Commons Attribution 4.0 License

Submission Link: https://biomedres.us/submit-manuscript.php
D3 Supplementation on Muscular Function in Older Adults with and Without Resistance Training: A Randomized, Double-blind, Controlled Study. J Gerontol a Biol Sci Med Sci 75(11): 2089-2097.

12. Wilkinson DJ, Hossain T, Hill DS, Phillips BE, Crossland H, et al. (2013) Effects of leucine and its metabolite $\beta$-hydroxy- $\beta$-methylbutyrate on human skeletal muscle protein metabolism. J Physiol 591(11): 29112923.

13. Chen S Y, Wu N, Lee YC, Zhao Y (2013) Association between muscle atrophy/weakness and health care costs and utilization among patients receiving total knee replacement surgery: a retrospective cohort study. Journal of pain research 6: 595-603.

14. Herzog MM, Marshall SW, Lund JL, Pate V, Spang JT, et al. (2017) Cost of Outpatient Arthroscopic Anterior Cruciate Ligament Reconstruction Among Commercially Insured Patients in the United States, 2005-2013. Orthop J Sports Med 5(1): 2325967116684776.

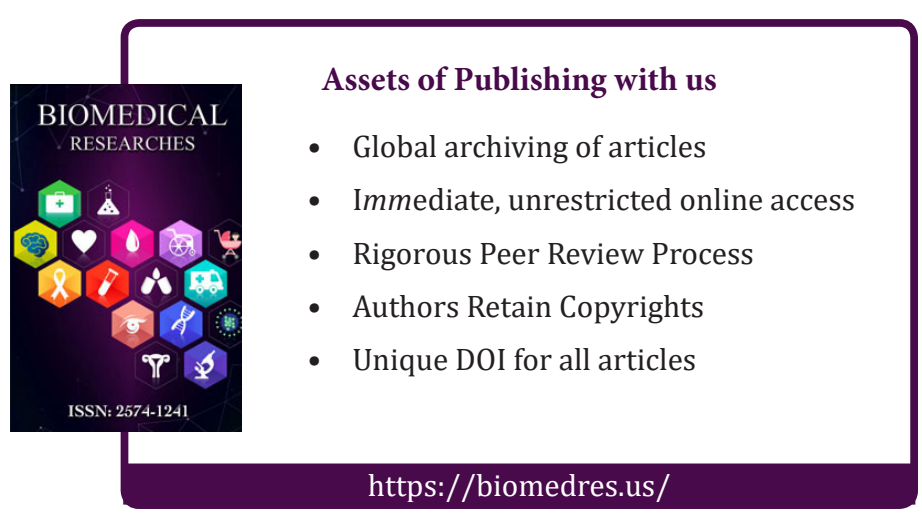

